\title{
Modelo de enseñanza-aprendizaje soportado en la malla del conocimiento
}

Eugenia Grosso Molano ${ }^{1}$

José Nelson Pérez Castillo²

\begin{abstract}
Resumen
Las comunicaciones generan nuevas posibilidades en el sector educativo al romper con las limitaciones geográficas y temporales. Se han creado aplicaciones relativamente sencillas que pueden ser reutilizadas por individuos o grupos dispersos geográficamente. La información relacionada con la malla computacional, e-learning, software educativo Sakai, gestión del conocimiento y manejo de estándares se debe tener en cuenta para desarrollar un trabajo colaborativo, empleando el modelo de aprendizaje TPCK que integra tres componentes principales que son: conocimiento- tecnología, conocimiento-pedagogía y conocimiento de contenidos curricular. La sumatoria equivale a la asignatura Introducción a la Programación, que emplea los objetos de aprendizaje de tal manera que permiten la reutilización de información.
\end{abstract}

Palabras clave: e-learning, modelo de aprendizaje TPCK, servicios web, gestión del conocimiento, mallas computacionales. 


\begin{abstract}
Communications create new opportunities in the education sector breaking down geographical and time limitations, creating relatively simple applications can be reused by individuals or groups of geographically dispersed. Information related to the computational mesh, e-learning, educational software, Sakai, knowledge management, management of standards should be taken into account in developing a collaborative work, by using the model of learning TPCK which integrates three main components namely: knowledge-technology, knowledge-pedagogy and curricular content knowledge. The result is the subject "Introduction to Programming", using learning objects that allow the reuse of information.
\end{abstract}

Key words: e-learning, TPCK learning model, web services, knowledge management, computing grids.

Recibido: 20 de septiembre de 2010

Aceptado: 20 de octubre de 2010

\title{
Introducción
}

Las tecnologías de la información y las comunicaciones (TIC) junto con el internet han permitido desarrollar e implementar nuevos modelos de enseñanzaaprendizaje, integrando componentes como: conocimiento tecnológico, conocimiento pedagógico y conocimiento de contenido, TPCK, todo ello basado en e-learning,33 en la creación de ambientes interactivos que permiten acceder, interactuar, trasmitir e intercambiar conocimiento en procesos de capacitación y entrenamiento, transformando diversas áreas del saber y facilitando el acceso a recursos y servicios de la malla computacional.

La malla computacional permite el uso compartido y coordinado de los recursos disponibles en la red entre individuos y/o instituciones que a menudo se conocen como "organización virtual". Los usuarios se conectan a ella a través de servicios web basados en estándares abiertos; junto al e-learning, todo ello permite mayor flexibilidad y conexión de los usuarios que se encuentran geográficamente dispersos. Precisamente, para poder acceder a estos recursos se hace necesario el manejo de estándares de Arquitectura Orientada a Servicios (SOA). La infraestructura generada está construida sobre la arquitectura Open Grid Services Interface (OGSA) de Globus. 
La malla computacional, junto con el conjunto de metodologías y herramientas dadas a través de un software educativo como Sakai, proporciona y abre un nuevo espacio cultural, educativo e, incluso, comercial, que promete revolucionar el mundo de la computación al enfocarse fundamentalmente en el acceso remoto a recursos computacionales sin la limitación de tener que centrarse en una sola tecnología concreta.

Un modelo de enseñanza-aprendizaje que utilice las nuevas tecnologías y cree ambientes interactivos para la capacitación, entrenamiento y desarrollo, señala importantes cambios en las formas de producir, almacenar, recuperar y transmitir información y generar conocimiento. La integración de todos estos elementos (malla computacional, modelo TPCK, e-learning y software Sakai) en el ámbito de la educación, es lo que intentaremos recopilar en el presente artículo.

\section{Concepto de malla computacional}

La malla computacional se enmarca dentro de la tecnología de computación distribuida que engloba conceptos como: sistemas operativos distribuidos, programación de multiprocesador, redes de ordenadores, computación paralela, redes de computadoras, seguridad y bases de datos. Esto permite compartir recursos entre grupos que no pertenecen a una misma organización de manera que todos los participantes obtengan una experiencia satisfactoria (en términos de rendimiento, seguridad y usabilidad, entre otros).

La malla computacional permite ampliar su potencial sin la necesidad de aumentar la capacidad de procesamiento ni almacenamiento. Maneja una estructura abierta con estándares que facilitan la interoperabilidad y que permiten administrar, definir y negociar el uso de los recursos o servicios computacionales de diferentes organizaciones virtuales dispersas geográficamente para obtener una supercomputadora.

\section{- Modelos educativos}

Son los patrones conceptuales que permiten esquematizar de forma clara y sintética los elementos de un programa que se utilizarán en el proceso enseñanzaaprendizaje. Los docentes deben tener una idea general de los programas de la asignatura, de cómo operan y cuáles son los elementos que desempeñan un papel determinante en un programa o en una planeación didáctica, mediante instrumentos innovadores soportados en la malla computacional, que promete revolucionar el mundo de la computación y el modo como se desarrollan las aplicaciones actualmente. 
Inicialmente en los salones de clase se manejó la pedagogía (P) y el conocimiento de contenidos (C). Cada uno de estos componentes se trabajó de manera independiente. Los diseños experimentales condujeron a la búsqueda de metodologías que integran estos componentes y se propagó la expresión conocimiento de contenido pedagógico, PCK (Shulman, 1986). Este incluía la representación y la formulación de conceptos y las técnicas pedagógicas, lo que hacía que el conocimiento fuera fácil o difícil de aprender.

A través del tiempo, las bases del conocimiento en la educación se han centrado en el contenido de conocimiento del profesor (Shulman, 1986; Veal \& Makinster). Los diseños experimentales en búsqueda de metodologías condujeron a integrar estos componentes y surgió la expresión Conocimiento de Contenidos Pedagógicos, PCK.

Luego se empezó a utilizar un nuevo componente, la tecnología (T) de manera "transparente" (Bruce \& Hogan, 1998). Hacia 1980 se incorporaron el hardware y el software en la educación y se convierte en un componente clave para el manejo del conocimiento del profesor, como requisito para poder aprender y aplicar en el proceso de enseñanza. Se comenzó a combinar la tecnología $(\mathrm{T})$ con el conocimiento de Contenidos (C) y Pedagogía (P), y con estos componentes se conformaron parejas entre las que se crean nuevas ideas como conocimientos tecnológicos y conocimiento de contenidos (TCK) y el conocimiento del maestro se transformó en aplicaciones tecnológicas. Posteriormente se combinó el conocimiento tecnológico con la pedagogía, (TPK) lo cual permitió soportar objetivos pedagógicos.

Luego se integraron los tres componentes: conocimiento tecnológico, conocimiento pedagógico y conocimiento de contenidos, TPCK. (Shulman, 1987,1 986; Bruce \& Levin, 1997; Dewey \& Bentley, 1949; Rosenblatt, 1978). Este modelo permite introducir nuevos conceptos y requiere una sensibilidad al desarrollar la relación dinámica, transaccional entre estos. Acá se incluyen técnicas o métodos usados en el salón de clase de acuerdo con su naturaleza y estrategias para el mejoramiento de los estudiantes en la construcción del conocimiento.

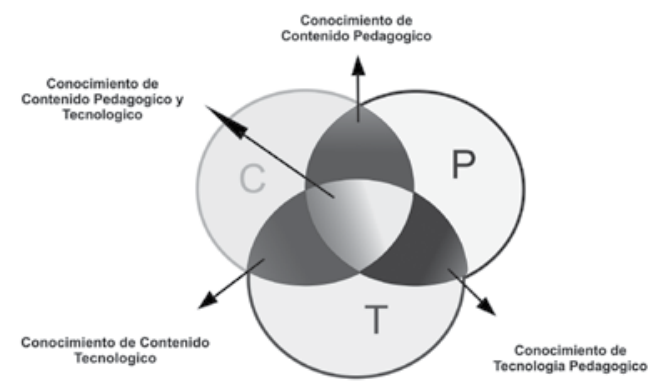

Figura 1. Estructura TPCK (Mishra, Koehler) 


\section{- E-learning}

Las tecnologías de la informática y las comunicaciones han permitido crear nuevas formas de aprendizaje, lo que rompió las limitaciones geográficas y temporales de los esquemas tradicionales de enseñanza-aprendizaje, al integrar diferentes recursos electrónicos en línea.

Al emplear e-learning se facilita el acceso a recursos y servicios permitiendo la distribución de contenidos educativos flexibles y adaptativos que puedan actualizarse rápidamente para luego ser publicados, $\mathrm{y}$ de esta manera se creen comunidades educativas que permitan el intercambio y la colaboración.

Una de las definiciones de e-learning es: "el software encargado de la gestión de usuarios, gestión de cursos y servicios de comunicación, proporcionando la oportunidad de crear ambientes de aprendizaje centrados en el estudiante." ${ }^{3} \mathrm{Se}$ puede decir que es el uso de tecnologías Internet para entregar un amplio rango de soluciones que mejoran el conocimiento y el rendimiento. El e-learning está basado en tres criterios fundamentales:

a) Trabaja en red, lo que lo hace capaz de ser instantáneamente actualizado, almacenado, recuperado y distribuido y permite compartir instrucciones o información.

b) Se entrega al usuario final a través del uso de ordenadores utilizando tecnología estándar de Internet.

c) Se enfoca en la visión más amplia del aprendizaje que va más allá de los paradigmas tradicionales de capacitación".

La implementación de las TIC ha permitido que el e-learning interactúe entre diferentes comunidades o usuarios debido a su fácil comunicación entre las personas.

Su acceso globalizado permite construir nuevo conocimiento colaborativo que se comparte, se comunica y se distribuye. Exige el replanteamiento de la enseñanza y el aprendizaje al incorporar herramientas que centren su conocimiento y experiencia en materiales didácticos, de tal manera que sean más asimilables por los estudiantes en forma colaborativa.

\section{Modelos de e-learning:}

Podemos considerar por sus características dos modelos de e-learning: sincrónico cuando el profesor y los alumnos al mismo tiempo se comunican directamente. 
El otro modelo es asincrónico, es decir cuando la comunicación no se realiza de manera simultánea.

\section{El modelo educativo virtual:}

En la modalidad basada en Internet, se definen los contenidos y actividades para un curso partiendo de la estrategia didáctica diseñada por el profesor. El alumno realiza su proceso de aprendizaje a partir de dichos contenidos y actividades, pero sobre todo, a través de su propia motivación por aprender, de la interacción con otros compañeros y de la guía y asesoría de su profesor.

Entre los estándares empleados en el manejo de e-learning se encuentran:

El Scorm (Sharable Content Object Reference Model): es una colección de estándares y especificaciones para el desarrollo, empaquetamiento y distribución de material educativo en cualquier parte del mundo, adaptados de múltiples fuentes para proporcionar un conjunto completo de capacidades de e-learning para productos de software que permiten la interoperabilidad, la accesibilidad y la reutilización de contenido de aprendizaje basados en web.

El IMS, (Instructional Management System, de Global Learning Consortium Inc., que organiza los objetos y recursos de aprendizaje de un curso y establece las pautas metodológicas en la distribución de los contenidos, soportando los procesos de comunicación entre los diferentes integrantes de acuerdo con su rol. El aprendizaje en la web requiere una interacción más rica y fiable ya que proporciona seguimiento al progreso del estudiante a través de múltiples cursos y modela las unidades de aprendizaje introduciendo la información en un paquete de contenidos, con la especificación del IMS Content Package (IMS CP).

La integración de Learning Management Systems (LMS) ofrece la notificación y agrega funcionalidad extra a través de las herramientas de comunicación, gestión, distribución, administración de usuarios, evaluación y seguridad. Luego se transforman en Learning Content Management Systems (LCMS) que es donde los desarrolladores pueden crear, almacenar, reutilizar y gestionar los contenidos digitales en diferentes niveles para luego reestructurar la información de manera dinámica, permitiendo crear objetos de aprendizaje de acuerdo con los requerimientos y estilos solicitados de un repositorio de objeto central.

Learning Technology Standards Committee (IEEE LTSC). Promueve la creación de normativas ampliamente aceptadas. El LTSC se encarga de preparar estándares abiertos acreditados, reportes y guías para el uso informático de componentes y sistemas de educación, como resultado de proyectos autorizados por IEEE Standards Association. 
PROmoting Multimedia Access to Education and Training in EUropean Society (PROMETEUS). Establece directrices, genera manuales de las mejores prácticas, proporciona recomendaciones a los organismos de normalización y a los responsables de políticas nacionales e internacionales. Su principal objetivo es apoyar el uso eficaz, la investigación, el desarrollo y la asimilación en el campo de las tecnologías del aprendizaje.

Advanced Distributed Learning(ADL). Promueve el acceso a la educación y capacitación de la más alta calidad, adaptada a las necesidades individuales. Involucra conceptos e ideas técnicas; hace prueba de especificaciones y estándares; trabaja en la elaboración de pautas para asegurar la interoperabilidad y reusabilidad de las tecnologías de e-learning que el gobierno puede adquirir en el futuro.

\section{Sakai}

Es una plataforma de libre distribución, que consiste en una comunidad de instituciones académicas en la que las organizaciones comerciales y los individuos trabajan juntos en un ambiente colaborativo que se adapta a las necesidades de los estudiantes, instructores e investigadores. La aplicación está diseñada para ser escalable, confiable, interoperable y extensible a través de Java, ya que es orientada a servicios. Es un sistema de administración y gestión de aprendizaje en línea, Learning Management System (LMS), que ofrece un espacio de trabajo en línea.

Tiene herramientas que realizan las siguientes acciones: administrar recursos, materiales y actividades de formación; gestionar evaluaciones; controlar y dar seguimiento al proceso de aprendizaje. También ofrece servicios de comunicación tales como: foros de discusión y videoconferencias, entre otros. Desde un enfoque más técnico, con Sakai también es posible administrar usuarios, brindar accesos y generar informes. Emplea otras herramientas provenientes de otros ámbitos para entregarlas a la vida académica: blogs, wiki, podcast, RSS, sistema de videoconferencia y mensajería instantánea.

\section{Gestión de conocimiento}

Se considera que el conocimiento es un "recurso crítico" que debe gestionarse de manera eficaz para contribuir a una mayor rentabilidad social y/o económica de las instituciones. Podríamos decir que "el conocimiento es la información combinada con la experiencia, el contexto, la interpretación y la reflexión, que es una clase de información de alto valor que está preparada para aplicarse a la toma de decisiones y a la realización de acciones."

En la actualidad, con la globalización, se necesita que las instituciones u organizaciones en donde los mercados, los productos, las tecnologías y las 
sociedades en su conjunto cambian a gran velocidad, gestionen su principal activo, el conocimiento, para que puedan producir más a menor costo y con mayor calidad.

Cuando hablamos de la gestión del conocimiento, implícitamente se están combinando tres conceptos:

- La valoración del know-how de las empresas, las patentes, las marcas de forma normalizada, es decir, el capital intelectual.

- El impulso de una cultura organizativa, orientada a compartir conocimiento y trabajo cooperativo.

- La puesta en marcha de dispositivos que faciliten la generación y el acceso al conocimiento que genera la organización.

El e-learning emplea la gestión del conocimiento para identificar, almacenar, crear, difundir y distribuir el conocimiento entre profesores, tutores y personal administrativo de una institución educativa.

La gestión del conocimiento se centra en la informática colaborativa y en el trabajo en equipo, de tal manera que facilite intercambio y colaboración entre las diferentes personas que intervienen en una institución educativa, al emplearse la tecnología, por ejemplo, en los trabajos en grupo (groupware), en la minería de datos (data mining), etc.

El trabajo en equipo se ve facilitado desde el mismo instante en que mejoran y se potencian los medios de comunicación disponibles entre los participantes en una actividad docente.

\section{Objetos distribuidos de aprendizaje:}

En un ambiente distribuido y propiciado inherentemente por la malla computacional, la movilidad es una característica esencial, de tal manera que a pesar de que los recursos que se encuentren dispersos entre los diferentes nodos, exista la posibilidad de disponer de ellos con naturalidad, haciendo "transparentes" los procesos de interoperabilidad. Esto no podría lograrse sin un esquema que vincule las redes, funciones esenciales de un modelo cognitivo, tal como lo sugiere la gráfica 1. Existen diferentes niveles de agentes en el modelo, ubicados tanto en la red de conocimiento como en la red de aprendizaje y en ciertos casos en la red de servicios Internet.

Se define como un conjunto de recursos digitales independientes, autocontenibles y reutilizables en diferentes contextos y plataformas, con un propósito educativo constituido por al menos tres componentes internos: contenidos, actividades de 
aprendizaje y elementos de contextualización. El objeto de aprendizaje debe tener una estructura de información externa (metadatos) que facilite su almacenamiento, identificación y recuperación.

Las características de una malla computacional determinan la disposición de los recursos en unidades digitales tales como videos, artículos, cuestionarios, fotografías, talleres, etc.

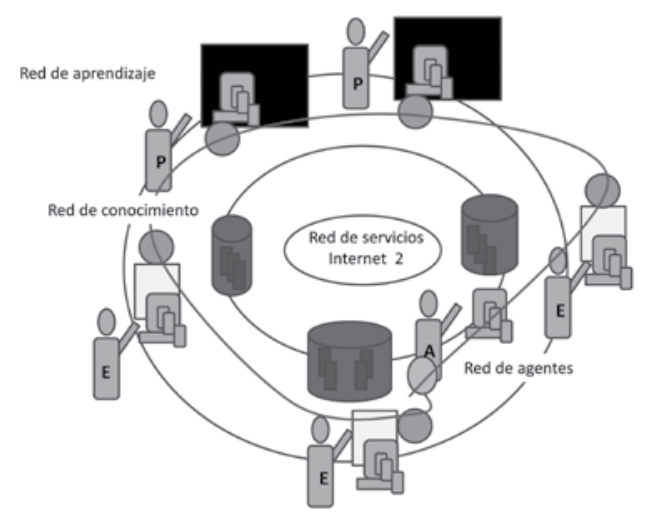

Figura 2. Modelo de educación basada en redes

Agente pedagógico

Los agentes pueden ser pedagógicos o sistemas tutores inteligentes. Estos agentes son utilizados en e-learning como tutores o compañeros de estudio que prestan asistencia a los procesos de aprendizaje con seres humanos en una variedad de dominios. Tienen la característica de ser activos pero no dominantes, actuar como colaboradores, competidores e incluso, como alborotadores o críticos de una persona. Por estas características, pueden ayudar a la labor del aprendizaje.

El agente podría aportar al estudiante contenidos, comunidades o actividades de cómputo específicas. Es necesario en este punto hacer uso de las técnicas de inteligencia artificial para poder razonar e inferir sobre las necesidades del estudiante. Estas características generalmente son manipuladas a través de entornos virtuales de aprendizaje como los portales.

\section{Conclusión}

La transformación de la educación con apoyo de las tecnologías de la informática y las comunicaciones se ha convertido en un proceso fundamental en el desarrollo de los procesos de enseñanza-aprendizaje. Empleando software educativo de 
código abierto como Sakai se pueden crear nuevas aplicaciones para múltiples usuarios en un entorno de trabajo colaborativo. La utilización de herramientas es una nueva forma de interacción entre el hombre y la tecnología informática y de compartir recursos a través de la malla computacional.

La nueva generación de tecnología ha transformado totalmente el papel social de la educación. El aprendizaje que solía ser un proceso humano, es ahora un proceso transhumano en el que la gente comparte cada vez con poderosas redes de conocimiento y logra ampliar la misma comunidad educativa al poder interactuar a través de los objetos de aprendizaje, con culturas y recursos heterogéneos. La presencia de éstos es transparente y garantiza el acceso de poblaciones excluidas del sistema educativo tradicional por limitaciones de tiempo y espacio.

Es evidente que la educación superior debe concentrar sus esfuerzos en el liderazgo de los programas de desarrollo de tecnología informática en la medida en que responda con eficacia a la necesidad de mejoramiento de los procesos educativos. Para ello debe emplear software educativo de código abierto que administre recursos, materiales y actividades de formación y que gestione, controle y dé seguimiento al proceso de aprendizaje.

\section{Referencias bibliográficas}

Baylor, A. Intelligent agents as cognitive tools for education. Educational technology. Camacho, R. Un sistema integrado, centrado en la grid para estudiar hidrodinámica del colapso gravitacional en la grid. Departamento de Física. (2007).

Casate Fernández, Ricardo. Gestión del conocimiento y del capital intelectual en el Centro de información y gestión tecnológica de la provincia de Granma, (CIGET) de Granma.

Castaño, J. C. \& Montoya, L. (2006). Un cuadro de mando para la gestión del conocimiento. Scientia et Technica año XII No 312006.

Castro, Harold. Grid Computing: Promesa de los sistemas distribuidos. Revista Sistemas ACIS, edición 98, octubre-diciembre 2006. Definición de aprendizaje significativo, en: http://www.psicopedagogia.com/definicion/aprendizaje\%2osignificativo. Acceso el 12 marzo de 2007.

Devedžić, Vladan. Web intelligence and artificial intelligence in education. Educational technology \& society. 2004.

Foix, Cristian, Zavando, Sonia. Estándares de e-learning. Centro de tecnologías de información. 2002. 
Globus Project. En http://www.globus.org. 2003. Report, Open grid service infrastructure WG.

Husemann, B., Lechtenb O., VOSSEN G., WESTERKAMP P. XLX - A platform for graduate-level exercises; in proc. int. conf. on computers in education, Auckland, New Zealand, diciembre de 2002.

IBM. Introduction to grid computing. IBM redbooks, en http://www.redbooks.ibm. com/abstracts/sg246778.html?

IMS learning design information model version 1.0, Final specification, IMS Global consortium. Revisión: 20 de enero de 2003. En: http://www.imsglobal.org/ learningdesign/Idv1po/imsld_infov1po.html\#1495294.

Itmazi, Jamil. Sistema flexible de gestión de e-learning para soportar el aprendizaje en las universidades tradicionales y abiertas. Tesis doctoral. Universidad de Granada, Granada, 2005.

Kesselman C., Foster I, Nick J, Tuecke S. The physiology of the grid: an open grid services architecture for distributed systems integration. Global grid forum. USA, 2002.

Korba, Larry, XU, Yuefei, Andrew, S., Patrick, Yee George, Ronggong, Song, Khalil ElKhatib. Privacy and trust in agent-supported distributed learning. Designing distributed environments with intelligent software agents. Idea group publishing, 2004.

Learning Consortium. Revisión: 20 de enero de 2003, en: http://www.imsglobal.org/ learningdesign/ldv1po/imsld_infov1po.html\#1495294.

Martin Laborda, Rocío. Las nuevas tecnologías en la educación. Fundación AUNA, 2005.

Ministerio de Educación Nacional. República de Colombia. En: http:// www. colombiaaprende.edu.co/html/directivos/1598/article-99393.html.

Mishra, P., \& Koehler, M. ¿What happens when teachers design educational technology? the development of technological pedagogical content knowledge. 2005.

Technological pedagogical content knowledge: a frame for teacher knowledge. Junio de 2006.

Mishra, Punya, Hershey, Kathryn, Shane, Cavanaugh. Teachers, learning theories, and technology, 2005.

Morales, Erla, García, Francisco J, Barrón, Ángela, Berlanga, Adriana, López, Clara. Propuesta de evaluación de objetos de aprendizaje. Instituto universitario de 


\section{Eugenia Grosso Molano y José Nelson Pérez Castillo}

Modelo de enseñanza-aprendizaje soportado en la malla del conocimiento, artículo de reflexión

ciencias de la educación, Universidad de Salamanca, Departamento de informática y automática, 2007.

Nájar Sánchez, Olga. Diseño y planificacion adaptativa de contenidos virtuales en plataformas CMS (Content Managemnt System) y LMS (Learning Management System) UPTC. Agosto de 2009.

Ortiz Medina, Janeth María. La educación virtual y el proceso cultural. En: http:// enredando.com.

Peña, I. Tesis Doctoral. intelligent agents to improve adaptivity in a web based learning enviroments, Universidad de Girona, 2004.

Richard M., Felder, Linda K, Silverman. Learning and teaching styles in engineering education.

Sánchez Arias, Víctor Germán. Diseño de un patrimonio de recursos educativos basado en una red de acervos abiertos y distribuidos de objetos de aprendizaje. Laboratorio nacional de informática avanzada A.C.

Señas, Perla. Aprendizaje basado en la web. Primeras jornadas de educación en informática y TICS en Argentina, 2005.

Smith, J., Yan, R. Ibm multimedia analysis and retrieval system 2009 [cited 2009 24/05/ o9]. En:http://alphaworks.ibm.com/tech/imars.

Stephens, Iva Angelina, Foronda Natalia, Trujillo John. Implementación de un estudio de caso usando objetos de aprendizaje (OA) para determinar la interoperabilidad entre diferentes plataformas e-learning.

Shulman, L. (1986).Those who understand: Knowledge growth in teaching. Education Researcher.

(1987). Knowledge growth in teaching: Foundation of the new reform. Harvard education review.

Unired. Segunda jornada de apropiación Unired-Renata. Recuperado el 23 de abril de 2009 desde: http://www.unired.edu.co/index.htm.

Valverde, Jesús, Garrido María del Carmen. Enseñar y aprender con tecnologías: un modelo teórico para las buenas prácticas con tics. Teoría de la educación y cultura en la sociedad de la información. Tesi, 2010.

Westerkamp, Peter. E-learning as a web service. University of Munster. 


\section{Revista de}

Wooldridge, Michael, Kinny Nicholas R., Jennings, David. The gaia methodology for agent-oriented analysis and design. kluwer academic publishers, 2000.

Wolf, Cristian, Weaver: Towards learning style-based e-learning in computer science education.

Yanko Ossandón Núñez, Objetos de aprendizaje: Un recurso pedagógico para e-learning.yossando@uta.2005.

Zhai, Yuqing, Qu Yuzhong y Ga, OZhiqiang. Agent-based modeling for virtual organizations in grid. International workshop on information grid and knowledge grid. 2004. 